\title{
Curricula Challenges in Computing / IT Disciplines in the Wake of Rapid Technological Advances, Changing Jobs Landscape, and Demands of Societal Needs
}

\author{
S.R. Subramanya ${ }^{1}$ \\ ${ }^{1}$ School of Engineering and Computing, National University, San Diego, USA.
}

\begin{tabular}{l} 
ARTICLE INFO \\
\hline Keywords: \\
advances in computing / \\
IT, automation, jobs \\
landscape, societal \\
problems, CS/IT \\
curricula
\end{tabular}

\begin{abstract}
The rapid advances in Computing, Communications, and Information Technologies have had tremendous impacts on various aspects of the lives of vast sections of humanity in such a short time. They have (a) created numerous kinds of new jobs, (b) made several other kinds of jobs to disappear due the kinds of automation they have created, and (c) transformed many professions based on their penetration in various facets of workflow. While these technological advances have resulted in many benefits to our society, they have also created many problems. Also, there are several other more prevalent problems in today's society than in the past, and the advances of technologies have yet to be put to effective use to solve them. These technological advances and their effects on our lives, on the jobs landscape, and the demands on these technologies to solve societal problems are expected to grow further in the near future. In the wake of these, from a higher education point of view, there are tremendous challenges in keeping the curricula in the Computing/IT disciplines up-to-date and relevant. In this paper, we will (a) briefly trace the major milestones in the developments of Computing, Communications, and Information Technologies, (b) give an overview of the current pressing societal problems, (c) present the projected trends in jobs landscape, and (d) outline some ways of keeping curricula in Computing/IT relevant in the wake of the challenges.
\end{abstract}

\section{Introduction}

There have been unprecedented advancements in Computing/IT, Communications, and Mobile technologies, and these are expected to continue. These technologies have, in addition, given rise to numerous other technologies. They have had tremendous impacts on almost all aspects of our lives by way of numerous products and services. As a formal discipline of study, Computing and related topics are only about 50 years old. In this short span of time, they have spawned numerous other sub-disciplines, several of which have gone on to become full-fledged disciplines by themselves. The Computing/IT technologies have reached significant levels of maturity and are

\footnotetext{
$\square$ Corresponding Author E-Mail Address: ssubramanya@nu.edu
} 
increasingly being used as the enablers (drivers) of almost all verticals such as manufacturing, transportation, retail, banking and finance, healthcare, energy and utilities, etc. as shown in Figure 1.

Figure 1: Several major segments driven by Computing, IT, and Mobile technologies

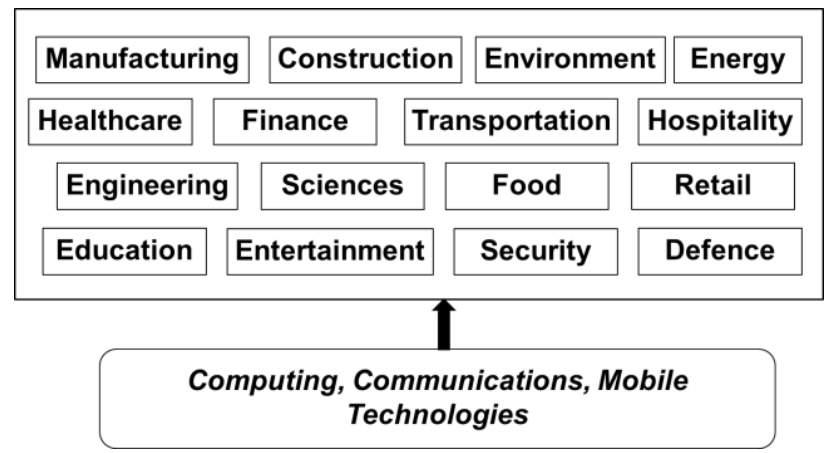

Computing/IT and related technologies have (a) created numerous kinds of new jobs, (b) made several other kinds of jobs to disappear due the kinds of automation they have created, and (c) transformed many professions based on their penetration in various facets of workflow. While these technological advances have resulted in many benefits to our society, they have also created many problems. A few examples are, cybercrimes, digital invasion of privacy, ills of social media, and several other problems which were nonexistent only a few years ago. Also, there are several other more prevalent problems in today's society than in the past, and the advances of technologies have yet to be put to effective use to solve them. These technological advances in Computing/IT, their effects on our lives, their impacts on the jobs landscape, and the demands placed on them to solve societal problems are expected to grow further in the near future. It is important for academicians, industry professionals, and policy makers to collaborate actively to come up with applications of these technologies for effectively solving the pressing and relevant societal problems. For higher education professionals it is important to keep the curricula in Computing/IT current and relevant.

In this paper, we will: (a) give an overview of some of the recent developments of Computing/IT and related technologies, (b) present an overview of the current pressing societal problems, (c) give the trends in jobs landscape, (d) outline the challenges in keeping curricula in Computing/IT relevant, and some ways of addressing those.

\section{Rapid technological advances and growth of data}

In recent times we have been witnessing the rapid growths in a plethora of technologies related to Computing/IT such as, Mobile technologies, Virtual Reality, Robotics, Quantum Computing, Autonomous Vehicles, Machine Learning and Artificial Intelligence, Cloud Computing, Big Data and Analytics, and Internet of Things, to name a few of the prominent ones, as shown in Fig. 2. Several of these have been put to use in varying degrees in the real-world applications. These have tremendous potentials in changing lifestyles of people and in improving the quality of life. 
Figure 2: Samples of emerging technologies

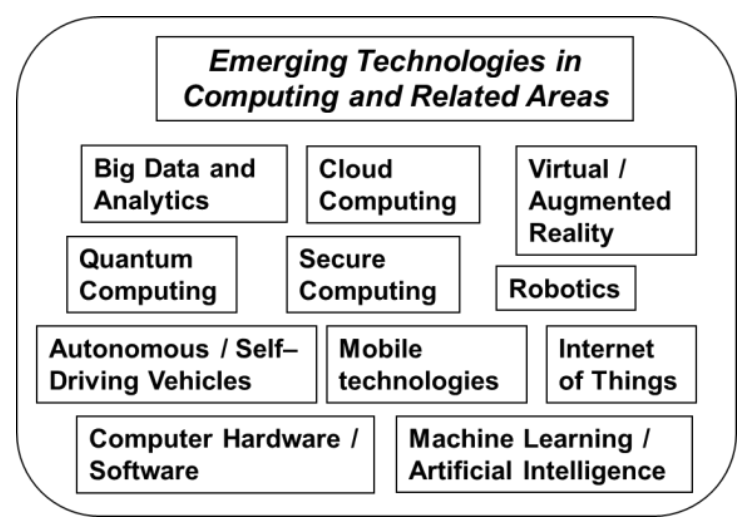

There have been several efforts in the past to explore alternatives to the von Neumann architecture. Quantum computing has received quite some attention in the recent past in this regard. Some recent breakthroughs include the announcement by IBM in March 2017 of the availability of API (Applications Programmer Interface) which enable developers and programmers to build interfaces between its existing 5-qubit cloud-based quantum computer and classical computers. Qubits are somewhat analogous to bits in a classical computer. Qubits can be in a 1 or 0 quantum state and can also be in a superposition of the 1 and 0 states. A 16-qubit universal quantum computing processor, and a prototype 17-qubit commercial processor was successfully built and tested by IBM in May 2017. [Wikipedia]

There have been numerous initiatives and also tremendous progress in autonomous driving vehicles. There are several trials where these vehicles have been driving in city streets. These can be especially beneficial to the elderly and disabled. However, there are numerous technical, policy, regulatory, and ethical issues that need to be addressed before they can become mainstream.

Computers (both traditional and embedded) used ubiquitously in almost all facets of our lives. With vast varieties of devices being connected to the Internet, and computers controlling infrastructure (both critical and non-critical) such as banking and finance, commerce, air travel, power and utilities, etc., the needs of security and privacy is extremely important than ever before. With increased complexity comes increased vulnerability. The numerous attacks on computers and breaches of databases worldwide have brought to the fore the criticality of security and privacy, making secure computing is an important research area.

The virtual reality (VR) and augmented reality (AR) technologies (also referred to as immersive technologies), have been transforming the way individuals interact with one another and with computing systems. Integration among mobile, wearable, Internet of Things (IoT) and sensor-rich environments are expected to extend immersive applications beyond isolated and single-person experiences.

The amount of data produced every day at present is about 2.5 quintillion (1018) bytes, and the rate of production of data is accelerating with increasing sources of data generation. About 90 percent of the data in the world today was generated in the last two years alone (Forbes, 2018). Big data generally refers to the huge volumes of data, generated from a variety of sources, at an accelerated rate, Some examples of the sources contributing to the big data are, Web logs, Social 
networks, RFID, Sensor networks, Internet text and documents, Internet search indexing, Retail, Call records, Astronomy, Remote sensing, Atmospheric science, Biological, Ecological, Genomics, Complex scientific data, Surveillance \& Public safety, Manufacturing, Military surveillance, Medical records, Photo and video archives (News/Entertainment), E-commerce, etc. This in turn has driven the needs for faster processing, enormous storage, high-bandwidth networks, novel user interfaces, etc. This is also giving impetus to data analytics and machine learning in order to automate the process of deriving meaningful insights, discoveries, and business intelligence from the huge amounts of data, which would otherwise be impossible/impractical by current techniques and methods.

\section{An overview of changing societal needs and current pressing societal problems}

There are numerous needs and challenges faced by the present-day society. Figure 2 portrays several of them - education, healthcare / public health, food and agriculture, water resources, environment, energy, transportation, public safety, disaster response/recovery/management, public administration / e-governance, community services, sustained development, and lifestyle management (Physical, Mental, Emotional, and Spiritual well-being). It requires innovative and interdisciplinary methods - drawing upon synergies from various (related) disciplines - in order to deploy the Computing/IT and related technologies for the effective and efficient solutions to the above problems.

Figure 3: Samples of pressing current societal issues

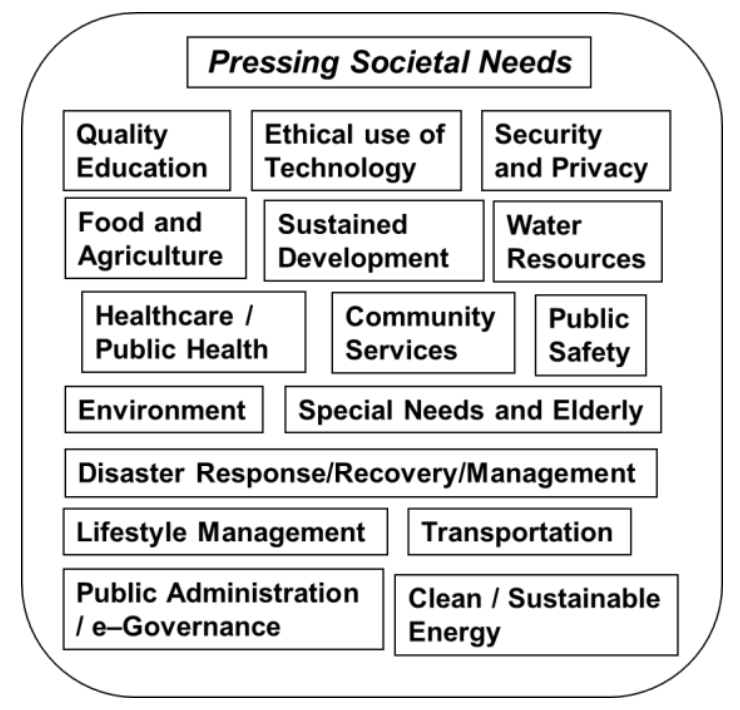

Quality education is very crucial for the sustenance of technological advancements and their effective applications for societal needs. There are several pressing issues in (higher) education. With changing technologies and landscape of jobs, it is important for (higher) education to be upto-date and relevant. 
With increases in population coupled with changing lifestyles, there have been demands to meet the needs and wants people. This has led, among many things, to increased industrialization, increased number of cars; increases in green-house gases, etc. There has been increasing demands for the energy. There is a critical need for clean and renewable energy sources.

The earth's ecology and environment is getting strained due to numerous ('man-made') factors such as, urbanization; industrialization; deforestation; pollutions of various kinds (water, air, noise, etc.), and several others. Existing technologies must be used and new technologies must be developed to address these issues.

The fast-paced technological and industrial developments place high demands on the resources - minerals, water, energy, chemicals, machinery, etc. They also produce several industrial waste - effluents, toxic gases, green-house gases, etc. The increasing population also produce lots of waste products and pollutants of numerous kinds. There is a crucial need for sustainable development.

Public safety comes to the fore in situations where there are lots of peoples in limited areas (i.e., the 'people density' is high). In normal day-to-day situations, examples of these are people at shopping malls, popular tourist attractions, airports, railway stations, office buildings, schools, etc. There are also "events" where it is not uncommon for people numbering 10,000 to over a Million converging at a single place (town/city) for public functions, cultural events, sports events (ex. National games, Olympics), religious events, etc. The safety and well-being of these people must be ensured by innovative proactive threat prediction and monitoring by novel applications of the technologies.

Several of the natural disasters (floods, cyclone, draught, etc.) seem to be periodical or seasonal (ex. floods, cyclones, hurricanes). Several of these can be predicted by using data (historic data, satellite date, sensor data, etc.), computer models, and simulations, and taking actions proactively. Some of them (ex. draught) could be avoided in several situations by the right understanding of the man-made conditions leading to such disasters, and addressing them proactively.

Almost all of the infrastructure are driven by Computing/IT. For example, the power grid, water supply and treatment, rail-road networks, air transportation, banking and finance, etc. Broadly, tangible/physical systems controlled by computers is referred to as cyber-physical systems. Any attack on the computer systems managing an infrastructure could have a disastrous effect, leading to crippling disruption in the services, loss of revenue, and added costs of recovery.

The seriousness of the cybercrimes and their increasing incidents can be gauged by noting that the cost to the global economy due to cybercrime (considering both direct and indirect costs) in 2016 was between $\$ 375$ and $\$ 575$ Billion, which was $15 \%-20 \%$ of the value of the Internet annual economy (in 2016) of $\$ 2-\$ 3$ Trillion. Thus the security aspects of the cyber-physical systems are very crucial.

\section{The changing landscape of jobs}

With technological advancements, several new kinds of jobs get created, several kinds of jobs vanish, and several others continue to exist, but with changing nature of the way they are done. Computing/IT, together with globalization, has been resulting in shifts in occupations and has generated studies in "21st century skills" which the students need to succeed in work (Trilling \& Fadel, 2009). The study in (Gallup, 2013), confirms the positive connection between the 
development of the competencies known as 21st century skills - critical thinking, real world problem solving, collaboration and communication skills - and future job success. In (Anderson \& Gantz, 2013) two of the requirements in a changing employment landscape which are listed are, increasing economic importance of digital commerce and digital content, and IT in the workplace. These require familiarity with technologies combined with analytical and problem-solving skills. A couple of examples of the changes brought about by technological advances are that the services sector employed $45.1 \%$ of the world's workers in 2013 and the share of services workers rose by $10.1 \%$ over two decades. The agricultural employment accounted for around one quarter, and saw a decline of $11.7 \%$ over the previous two decades (ILO, 2014).

The advancements in electronics, computers, and robots, have resulted in increased automation and control of numerous facets of manufacturing industries. Although this resulted in the reduction of some kinds of jobs/workforce, there have been increases in manufacturing jobs of different kinds. There will be increasing needs for people with skills in the maintenance of machines and systems driven by hardware and software. Recent advances in artificial intelligence, machine learning, knowledge management, and data analytics are expected to considerably reduce, if not completely eliminate, several while-collar workforce involved in routine and repetitive tasks. Automation driven by machine learning are increasing used as 'front-ends' in customer service, call center, tech help, travel agents, etc. With increased used of software as enablers in almost all verticals such as manufacturing, transportation, retail, banking and finance, healthcare, energy and utilities, etc., there will be increasing needs for people in software design, development, and maintenance. In general, highly specialized skills will be in demand, as demonstrated by the current emerging areas.

As with many technologies, Computing/IT also has a variety of layers in the workforce with different types of skills and competencies. We identify a five-layer pyramid model of human capital engagement, as shown in Figure 4.

Figure 4: Human Capital Engagement

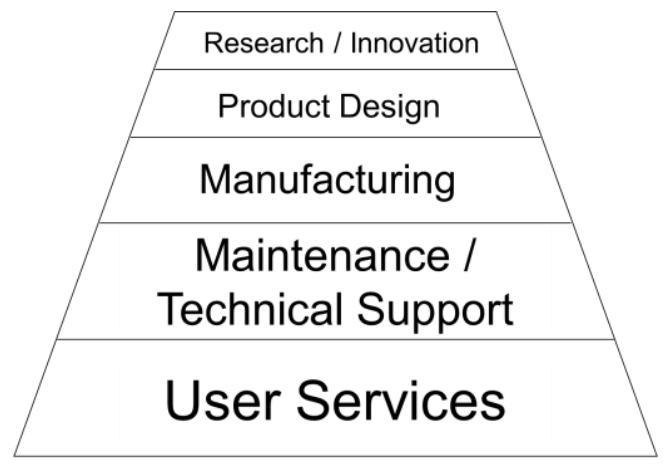

Innovation, at the top of the pyramid, requires high intellectual potential and develops cuttingedge technologies. The next layer below it is the product design, which is concerned with building the 'laboratory grown' proof of concepts into working models. The next layer is concerned with the actual manufacturing of products. The maintenance and technical support deals with the routine maintenance, trouble shooting, and services required to ensure smooth operations of the products and systems. User services is closely tied to maintenance and technical support, but having more 
customer-facing aspects than technical aspects. The relative sizes of the layers (although not to scale) are indicative of the fractions of the workforce expected to be working in these areas.

\section{Challenges posed by Computing/IT with regard to products / applications}

There are numerous challenges posed by the inherent nature of computing/IT technologies with regard to products, applications, educating and training manpower, etc. These technologies have a high rate of obsolescence due to the extremely high rate (almost exponential) of advancements. These technologies need to have a high rate of scale-up since they affect numerous domains and affect vast sections of humanity. In computing/IT, there are technologies which deal with dimensions as low as 10-9 and as high as 1018. Large and complex systems (hardware/software) pose challenges of reliability, backward compatibility, and support of legacy systems. These are summarized in figure 5 below.

\section{Figure 5: Challenges posed by the natures of Computing/IT technologies}

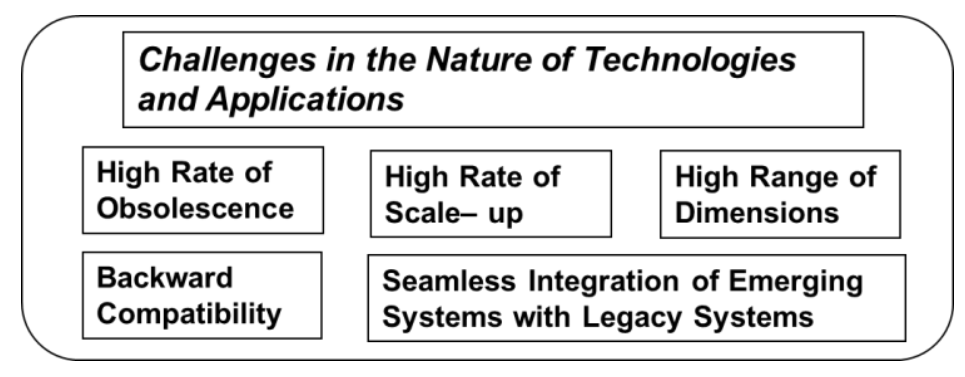

\section{Curricula challenges}

From a higher education perspective, it is important to keep the curricula in Computing/IT disciplines to be relevant and current, in order to (a) sustain the technological developments; (b) ensure a steady supply of qualified graduates to the industry/workforce; (c) enable the effective applications of technologies for pressing societal issues and needs. The importance of incorporating ICMT (information/computing, communications, and mobile technologies) in the early high-school and college curricula, and the methodologies of doing so are given in (Subramanya, 2014).

A few of the main high-level issues that need to be considered in the higher education of Computing/IT disciplines are shown in Figure 6.

Figure 6: A few of the major high-level issues to be considered in Computing/IT disciplines

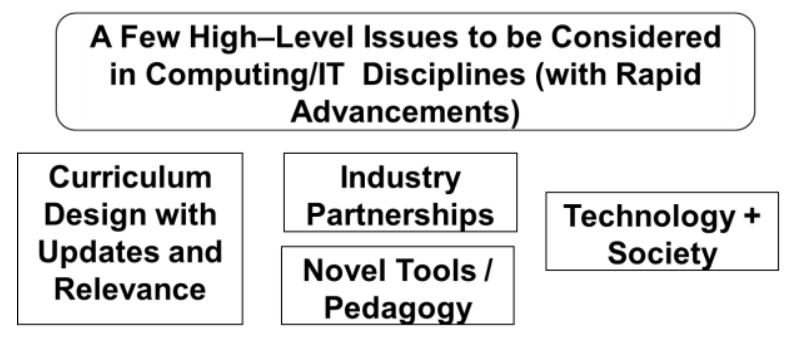


Careful design of curricula and frequent updates in order to maintain relevance, since the Computing/IT technologies change rapidly. Closer academia industry partnerships will facilitate keeping the curricula current and relevant and to make the students 'industry-ready'. Incorporation of novel tools and pedagogy will help in increasing the effectiveness of teaching and learning, and imparting the much needed skill sets to students. Last, but not least, the technologies must be put to use for addressing the needs and issues, and for the betterment of society.

\section{$\bigcirc$ Driving forces of curricula design}

A few major driving forces in designing the curricula for disciplines related to Computing/IT are shown in Fig. 7. A brief description of how the curricula has kept up with some of these and some suggestions about further updates to curricula are described below.

\section{Figure 7: Driving forces of curricula design}

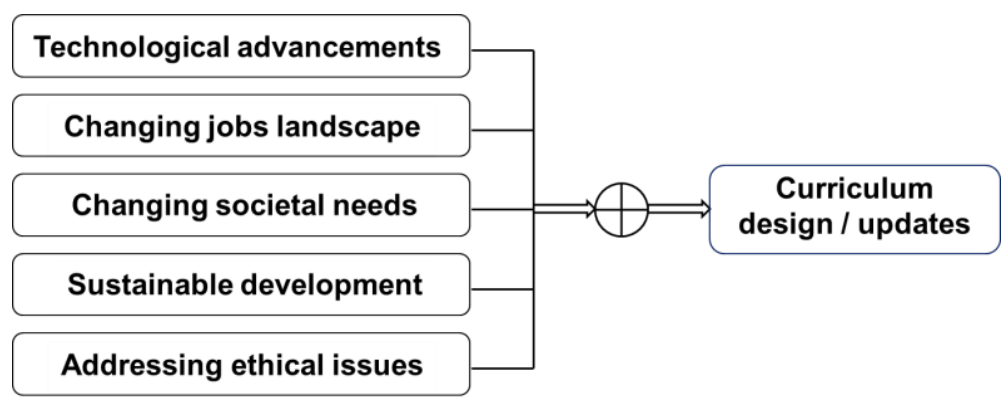

As a simple example how the curricula need to be redesigned frequently based on the rapidly changing technologies, consider the teaching of programming. We have witnessed the changes in the use of the mainstream introductory programming language, with the list (in chronological order) consisting of BASIC, FORTRAN, Pascal, Ada, C, C++, Java, Python. A course such as Numerical Analysis which was a mainstream course earlier is no longer a core course. A course on User Interface has taken on an important role considering the interactive nature of numerous software (including OS) and increased use of mobile platforms.

With computing systems increasingly used as enablers and drivers of numerous systems (embedded, cyber-physical, infrastructure, etc.), the security of those systems is becoming very critical. Thus incorporation in the Computing/IT curricula of courses in several aspects of security (ex. Cryptography, Network security, Web security, Security protocols, Mobile/Wireless security, etc.), as well as incorporation of security aspects in several courses (ex. Programming Languages, Operating Systems, etc.) is important.

The landscape of jobs has been changing rather frequently (compared to a few decades ago). The kinds of courses in the program of study should be relevant in terms of preparing the students to the available jobs. In recent times, cloud computing, big data and analytics, machine learning, secure computing, mobile computing, etc., have matured and have created job markets for the above skills. Although there have been updates to curricula reflecting some/several aspects of the above trends, there is need for more updates, without compromising several fundamentals.

In today's accelerated pace of technological developments, increasing globalization and competitiveness, and changing perception of societal/human values, there is are dire and serious needs for students to have a holistic picture, in addition to fundamental and specialized technical courses. One solution is to incorporate in the curricula (a) elements of sustainable development, 
(b) role of technology in society, and (c) ethical issues in the development, deployment, and use of technologies.

\section{- Recommendations for keeping CS/IT curricula updated and relevant in the wake of the challenges}

In the wake of technological advances, changing needs of society, changing jobs landscape, we present a layered approach shown in Fig. 8 to maintain the curricula in Computing/IT disciplines current and relevant.

Figure 8: Different layers in the design of curricula of Computing/IT

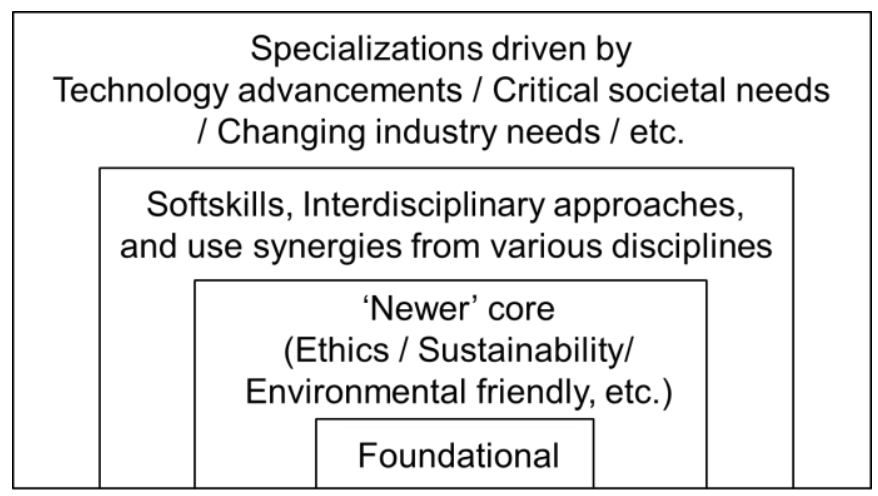

The descriptions of the layers are given below.

A. Technical foundations. Provide strong technical foundations of basic principles and techniques of Computing/IT, taking into consideration the newer technologies and techniques.

B. Extension to core. Provide extensions to the core by incorporate appropriate pressing issues of the society, since the ultimate use of technology is the betterment of the society.

C. Interdisciplinary approaches. Have emphasis on interdisciplinary approaches use of synergies across disciplines. Incorporate skills for handling complexity and use of modern (software / analytical) tools for effectively solving information-rich, information-driven, realworld problems. and making a strong connection to real-world applications

D. Newer specializations. Since many new (sub) areas of Computing/IT have been growing, design and implement appropriate and relevant specializations, driven by critical needs and importance to societal needs.

\section{Conclusion}

The fact-paced developments in Computing/IT and related technologies are beginning to have tremendous impacts on our lives. In order to sustain these developments and to put them to proper use, there are critical needs for developing policies and guidelines for the uses and applications of technologies. There are several pressing current societal problems which call for innovative applications of the Computing/IT technologies. There have been drastic changes to the landscapes of the workforce in several areas due to rapid advances in Computing/IT technologies. From the perspective of higher education institutions, keeping the curricula in Computing/IT disciplines is challenging. This paper presented some of the major milestones in the developments of Computing/IT related Technologies, gave an overview of the current pressing societal problems, 
presented the projected trends in jobs landscape, and outlined some ways of keeping curricula in Computing/IT relevant.

\section{References}

Wikipedia. https://en.wikipedia.org/wiki/Quantum_computing

Forbes report. (2018). https://www.forbes.com/sites/bernardmarr/2018/05/21/how-much-data-dowe-create-every-day-the-mind-blowing-stats-everyone-should-read/\#52d1928860ba

Trilling, B. and Fadel, C. (2009). 21 st Century Skills - Learning for Life in our Times, John Wiley.

Gallup report. (2013) "21st Century Skills and the Workplace: A 2013 Microsoft Partners in Learning and Pearson Foundation Study”, 2013.

Anderson, C. and Gantz, J.F. (2013). "Requirements for Tomorrow's Best Jobs - Helping Educators Provide Students with Skills and Tools they Need", IDC White Paper.

ILO report. (2014). Global Employment Trends, International Labor Organization Report, (ISBN:978-92-2-127486-5).

Subramanya, S.R. (2014). "Building Skills for the Effective Uses of Information, Communications, and Mobile Technologies, and their Applications to Societal Needs", Proc. National Conf. on Recent Innovations in Science, Engineering \& Technology(NCRISET), Pune, India, pp66-69. 